\title{
Indexing Fingerprints using Minutiae Quadruplets
}

\author{
Ogechukwu Iloanusi \\ University of Nigeria, Nsukka \\ oniloanusi@gmail.com
}

\author{
Aglika Gyaourova and Arun Ross \\ West Virginia University \\ http://www.csee.wvu.edu/ ross
}

\begin{abstract}
The computational complexity of matching an input fingerprint against every entry in a large-scale fingerprint database can be prohibitive. In fingerprint indexing, a small set of candidate fingerprints is selected from the database and only images in this set are compared against the input probe fingerprint thereby avoiding an exhaustive matching process. In this paper, a new structure named "minutiae quadruplet" is proposed for indexing fingerprints and is used in combination with a clustering technique to filter a fingerprint database. The proposed indexing algorithm is evaluated on all datasets in the Fingerprint Verification Competition (FVC) 2000, 2002 and 2004 databases. The high hit rates achieved at low penetration rates suggest that the proposed algorithm is beneficial for indexing. Indeed, it was observed that for $50 \%$ of the fingerprints, in most of the datasets, the penetration rate was less than $5.5 \%$ at a $100 \%$ hit rate. The robust performance across different databases suggests that the indexing algorithm can be adapted for use in large-scale databases.
\end{abstract}

\section{Introduction}

Two problems associated with human identification in large-scale fingerprint databases are (a) a long response time due to high computational complexity, and (b) a potential increase in false matches with increasing database size. Approaches taken to solve these problems include fingerprint classification, sub-classification and indexing [1]. Fingerprint indexing refers to the assignment of a numerical index vector to a fingerprint. The indexing mechanism includes a retrieval strategy that is invoked for selecting candidate fingerprints from the database (or gallery) based on their similarity with the input probe in the index vector space. The selected fingerprints constitute the candidate list of fingerprints for the given probe. By limiting the matching operation to those fingerprints in the candidate list and eliminating the need for exhaustive matching of the probe with every fingerprint in the gallery, indexing methods are able to reduce the response time in large-scale fingerprint databases.
In the literature, techniques employed for fingerprint indexing are based on ridge features, ridge pattern or structures derived from ridge features [3,4], minutiae features $[5,6,7]$, a combination of features $[2,8]$ or matching scores [9].

One of the earliest techniques for indexing fingerprints based on minutiae triplets [5] uses features such as sides of the triangle and minutiae orientation that are highly sensitive to fingerprint distortion. This feature set was improved in [6] but with the additional cost of introducing multiple thresholds that had to be trained on the images in the gallery.

In this work, a new topological structure based on minutiae quadruplets is proposed for indexing fingerprints. A minutia quadruplet is a quadrilateral formed from a set of 4 minutiae points as shown in Figure 1. Compared to minutiae triplets, minutiae quadruplets allow for the use of features that are less sensitive to distortion.

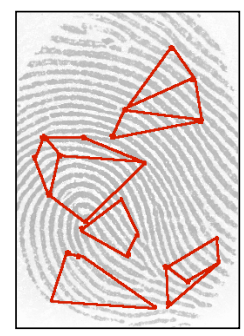

Figure 1: Sample minutiae quadruplets in a fingerprint image.

\section{Features for Indexing}

Given a quadrilateral, several different geometric features can be extracted. Initially, a set of 17 different features was considered. Based on a systematic evaluation process, some of these features were eliminated. In this work, seven features, $F=\left\{\varphi_{1}, \varphi_{2}, \delta_{1}, \delta_{2}, \rho_{1}, \rho_{2}, \eta\right\}$, from a minutiae quadruplet are proposed for indexing fingerprints. The geometrical interpretation of $\delta_{1}, \delta_{2}, \rho_{1}$, and $\rho_{2}$ is shown in Figure 2. 


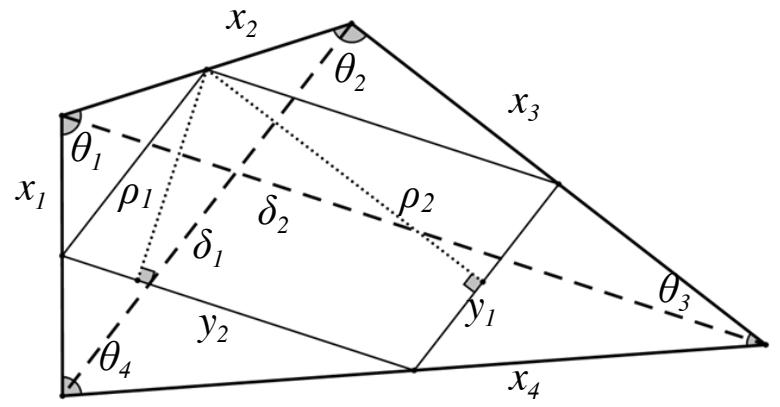

Figure 2: A minutiae quadruplet

\subsection{Difference of Internal Angles}

The first two features, $\varphi_{1}$ and $\varphi_{2}$, are the differences of two opposite angles in the quadruplet:

$$
\begin{aligned}
& \varphi_{1}=\theta_{1}-\theta_{3} \\
& \varphi_{2}=\theta_{2}-\theta_{4},
\end{aligned}
$$

where $\theta_{1}, \theta_{2}, \theta_{3}$ and $\theta_{4}$ are the four internal angles of the quadruplet. These differences are more robust to distortion compared to the interior angles themselves. When one of the vertices changes its position due to distortion in the fingerprint image, two of the interior angles change in a similar manner and, therefore, their difference may change by a very small amount or may even remain unchanged.

\subsection{Diagonals of the Quadruplet}

The second pair of features, $\delta_{1}$ and $\delta_{2}$, are the diagonals of the quadruplet. The two diagonals, $\delta_{1}$ and $\delta_{2}$, can tolerate distortions due to one or two minutiae points. If a single point or two opposite points are distorted, the length of the diagonal opposite to these points does not change. Figure 3 shows an example where two opposite minutiae points, $\theta_{1}$ and $\theta_{3}$, have changed positions and the length of the diagonal $\delta_{1}$ remains the same.

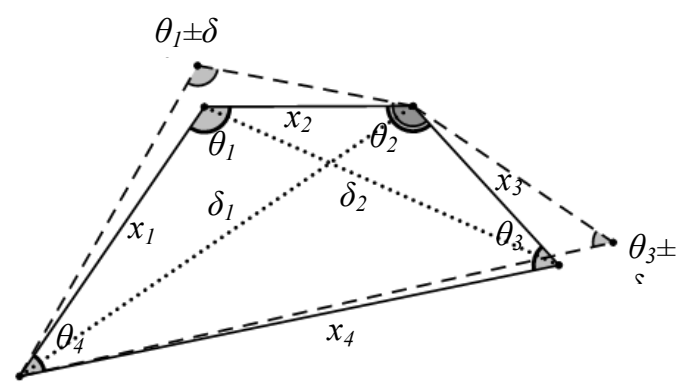

Figure 3: Change in the position of one or two opposite vertices does not affect the length of the diagonal connecting the other two vertices. In this figure, the length of diagonal $\delta_{1}$ stays the same.

\subsection{Heights of the Parallelogram}

The next pair of features, $\rho_{1}$ and $\rho_{2}$, are the heights of the inner parallelogram, whose vertices are the midpoints of the sides of the quadruplet.

\subsection{Global Feature $\eta$}

The last feature is a composite global feature that combines the sides and the areas of the quadruplet and the parallelogram:

$$
\eta=100 \log _{10}(\tau v) \text {, }
$$

where

$$
\tau=\sqrt{A_{P}}+\sqrt[4]{x_{1} \times x_{2} \times x_{3} \times x_{4}}
$$

and

$$
v=\sqrt{A_{q}}+\sqrt{y_{1} \times y_{2}}
$$

$A_{p}$ is the area of the parallelogram, $x_{1}, x_{2}, x_{3}$ and $x_{4}$ are the lengths of the sides of the quadruplet, $A_{q}$ is the area of the quadruplet, and $y_{1}$ and $y_{2}$ are the lengths of the sides of the parallelogram.

The heights of the parallelogram, $\rho_{1}$ and $\rho_{2}$, together with the lengths of the diagonals of the quadruplet define the general shape of the quadruplet. Therefore, $\eta$ is a general description of the shape and the size of the quadruplet and is only slightly affected by minutiae distortions.

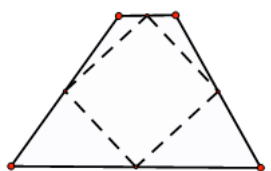

(a) Area $=15.01 \mathrm{~cm}^{2}$ $\eta=163.04$

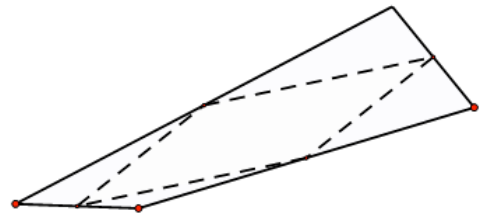

(c) Area $=20.1 \mathrm{~cm}^{2}$ $\eta=189.59$

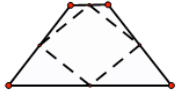

(b) Area $=7.01 \mathrm{~cm}^{2}$ $\eta=131.02$

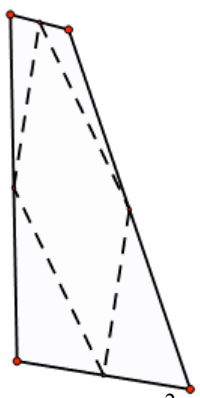

(d) Area $=20.1 \mathrm{~cm}^{2}$ $\eta=181.56$
Figure 4: (a) and (b) are quadruplets with similar shapes but different sizes, while (c) and (d) are quadruplets with different shapes but same sizes. 
Figure 4 shows four examples in which the quadruplets are drawn to scale. The quadruplets in Figure 4 (a) and (b) have similar shapes but different sizes and their $\eta$ varies according to their respective sizes. In Figure 4 (c) and (d), the quadruplets are very different - though they have the same size (quadruplet area $=20.1 \mathrm{~cm}^{2}$ ), the values of $\eta$ are different.

As shown in our experiments, the global feature, $\eta$, can be used as a single feature for indexing leading to relatively good results.

\subsection{Types of Quadruplets}

Three types of irregular quadruplets, i.e., convex, concave and reflex (crossed), can be formed from four vertex points as shown in Figure 5. Concave quadruplets were discarded and not used in the indexing experiments while all reflex quadruplets were converted to convex.

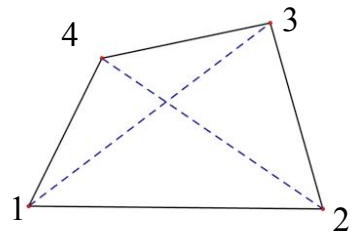

(a)

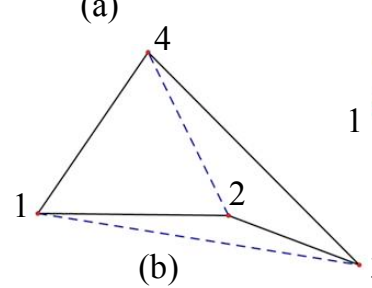

(b)

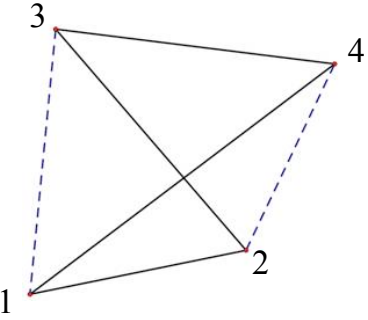

(c)
Figure 5: Irregular quadruplets. The sides are indicated by solid lines and the diagonals are indicated by dashed lines. (a) Convex. (b) Concave. (c) Reflex.

\section{Proposed Indexing Algorithm}

In the proposed approach, each fingerprint image is viewed as a set of quadruplets with each quadruplet being represented by the 7 aforementioned features. In the discussion below, the term feature vector is used to denote the 7 features pertaining to a single quadruplet. First, we use a training set of fingerprint images to construct an index space. The feature vectors extracted from all these images are clustered using the k-means algorithm [10]. The centroids of the clusters, $c_{1}, c_{2}, \ldots c_{k}$, computed using the arithmetic mean, define the index space.

An indexing string is computed for each image in the gallery and stored as a row in a table $\mathbf{T}$. The length of the indexing string is the number of clusters in the index space. Thus, each column in $\mathbf{T}$ corresponds to a cluster and each row corresponds to an image from the gallery.

To construct the indexing string of an input image, its quadruplets $\left\{q_{w} \mid w=1,2, \ldots Q\right\}$, are assigned to the clusters generated in the index space using the minimum distance rule based on the Euclidean distance:

Assign $q_{w}$ to $c_{k}$, if $k=\arg \min \left\{l_{2}\left(q_{w}, n_{j}\right), j=1 . . k\right\}(6)$

Here, $n_{j}$ is the centroid of the $\mathrm{j}^{\text {th }}$ cluster, $l_{2}$ is the Euclidean distance and $c_{k}$ is the cluster id. Each quadruplet is assigned to a single cluster. The indexing string is constructed by counting the number of quadruplets assigned to each cluster. Thus, the indexing string of image $x$ is $S(x)=\left\{a_{1}^{x}, a_{2}^{x}, \ldots, a_{k}^{x}\right\}$ where $a_{i}^{x}$ is the number of quadruplets from image $x$ which are assigned to cluster $i$, and $k$ is the total number of clusters. We will refer to $a_{i}$ as an accumulator for centroid $i$.

\section{Algorithm for indexing an input image}

1. Let $x$ be the input image, $\left\{q_{w} \mid w=1 \ldots Q\right\}$ be the quadruplets extracted from $X$, and $\left\{a_{i}, i=1 \ldots k\right\}$ be the accumulators for each centroid.

2. Set the accumulators for all centroids to zero.

3. For each $q_{w}$

Find the closest centroid $k$ using (6)

Increment the accumulator for $k$, e.g., $a_{k}=a_{k}+1$.

4. Construct an indexing string $S(x)=\left\{a_{1}^{x}, a_{2}^{x}, \ldots, a_{k}^{x}\right\}$.

5. Insert $S(x)$ and the id of $x$ into $\mathbf{T}$.

During retrieval, an indexing string is created for the probe image. Next, a sorted list of clusters in descending order of the corresponding accumulators is generated. The cluster id on the top of the list corresponds to that cluster which was assigned the largest number of quadruplets from the probe. This sorted list is used to identify a small number of clusters. Specifically, the clusters in the sorted list that were assigned $60 \%$ of the quadruplets from the probe are considered.

Once the desired clusters are identified, the corresponding accumulators from table $\mathbf{T}$ are used as votes for the gallery image they belong to. The top $M$ gallery images having the largest number of votes constitute the candidate list.

Table 1: Databases used to create the index space for each evaluation experiment.

\begin{tabular}{|l|l|}
\hline $\begin{array}{l}\text { Database used to } \\
\text { create the index } \\
\text { space }\end{array}$ & Database used for evaluation \\
\hline FVC 2000 DB1 & $\begin{array}{l}\text { FVC 2000 DB3, FVC 2004 DB1, } \\
\text { FVC 2002 DB1 and DB2 }\end{array}$ \\
\hline FVC 2000 DB2 & FVC 2002 DB3 \\
\hline FVC 2002 DB2 & FVC 2000 DB1 and DB2 \\
\hline FVC 2000 DB4 & FVC 2002 DB4 \\
\hline FVC 2002 DB4 & FVC 2004 DB4, FVC 2000 DB4 \\
\hline
\end{tabular}




\section{Algorithm for retrieving candidate images}

Let $S(x)=\left\{a_{1}, a_{2}, \ldots, a_{k}\right\}$ be the index string of the probe $p, Q$ be the number of quadruplets in the probe and $\mathbf{T}_{\mathrm{G} k \mathrm{k}}$ be the table storing the accumulators for the images in the gallery.

1. Sort $S(p)$ in descending order resulting in a sorted list $\left\{a_{i l}, a_{i 2}, \ldots, a_{i k}\right\}$.

2. Find the top $r$ clusters of the probe that contain at least $60 \%$ of the quadruplets.

3. For each gallery image $g$ sum the accumulators in $\mathbf{T}$ corresponding to the $r$ clusters. Let the sum be $C^{g}$.

4. Sort $C^{g}$ for $g=1 \ldots G$ in descending order.

5. Retrieve the gallery images corresponding to the top $n$ $C^{g}$ values.

Step 2 in the retrieval algorithm greatly reduces the number of clusters considered during retrieval, i.e., $r \leq 7$ for most cases. Furthermore, not every identity is assigned to every cluster and, therefore, many of the $C^{g}$ sums will be equal to zero. An efficient implementation of Step 4 should discard all identities for which $C^{g}=0$, prior to sorting.

\section{Experiments}

\subsection{Experimental Setup}

The databases used for the experiment were the Fingerprint Verification Databases (FVC) 2000, 2002 and $2004[11,12,13]$ each of which has four datasets - DB1A, DB2A, DB3A and DB4A. Each dataset contains 8 images for each of 100 subjects making a total of 800 images.

The dataset used to create the index space was different from the dataset used for evaluating the proposed scheme. Images of the first 25 subjects in a dataset were used for creating the index space. For each indexing experiment, the dataset used for creating the index space was chosen based on the following criteria: the image resolution was the same as the dataset used for evaluation and the scanners had similar properties. Five different index spaces were created for the 12 databases used in the indexing experiments, as shown in Table 1.

For each subject, 4 impressions were placed in the gallery while the remaining 4 impressions were used as probes. The 4 gallery impressions for a subject were selected at random. The VeriFinger SDK was used to extract minutiae points from the images.

For images containing a large number of minutiae points, the number of quadruplets may be prohibitively large. Therefore, the number of quadruplets for each image used in the experiments was empirically limited to
1200 by removing the largest quadruplets. This was done by removing the quadruplets having a diagonal larger than a threshold until the number of the remaining quadruplets reached 1200. Furthermore, concave quadruplets were not used and all reflex quadruplets were converted to convex. Reducing the number of the quadruplets is performed offline for the images in the gallery.

The number of clusters, $k$, used in the experiments was 50 for each dataset. This number was chosen empirically as a compromise between high penetration rates (for $\mathrm{k} \sim 30$ ) and low hit rates for $(\mathrm{k} \sim 100)$.

\subsection{Evaluation of Indexing Performance}

Indexing performance can be measured using two factors: the hit rate and the penetration rate. The hit rate denotes the fraction of probes for which the selected candidate list contains the correct identity, and the penetration rate denotes the average length of the candidate list retrieved for each probe.

\section{Results}

The results of the experiments on the 12 databases are reported in Figures 6, 7 and 8. For each experiment, the following three scenarios were considered: datasets in their original form; datasets with $20 \%$ spurious minutiae; and datasets with $20 \%$ missing minutiae. In each plot, the labels ending with $\mathrm{N}, \mathrm{M}$ and $\mathrm{S}$, represent the original, the missing and the spurious minutiae sets, respectively.

An important result of the proposed approach is the consistently low penetration rate at a hit rate of $100 \%$ which varies from $18.25 \%$ to $35.25 \%$ in the original minutiae sets, from $24.50 \%$ to $39.00 \%$ in the $20 \%$ missing minutiae sets, and from $34.75 \%$ to $57.5 \%$ for the $20 \%$ spurious minutiae sets.

Furthermore, deleting $20 \%$ of the minutiae points did not degrade the performance substantially. This shows that the minutiae quadruplets are robust to low quality images.

The results of the experiments with spurious minutiae are acceptable despite the generous quantity of randomly generated spurious data that were added to all the fingerprints. This shows that the minutiae quadruplet features are robust to a certain degree of noise in the fingerprints.

Finally, the results are similar across all databases showing the robustness of the proposed technique to different scanners.

An experiment in which the global feature, $\eta$, alone was used for indexing, led to penetration rates of $12.25 \%$ and $38 \%$ at $80 \%$ and $100 \%$ hit rates, respectively, on FVC 2000 DB1. 

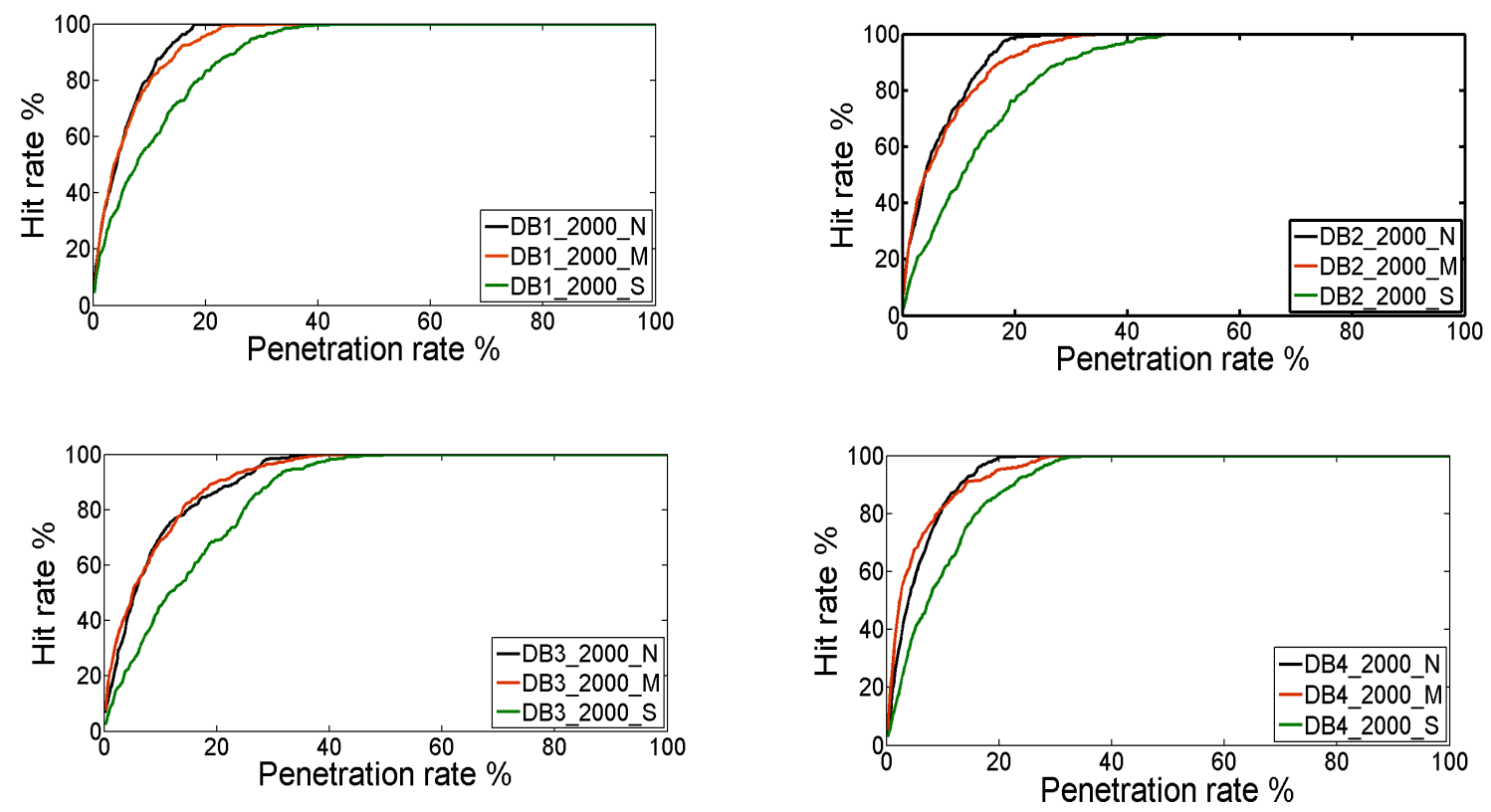

Figure 6: Performance on FVC 2000 DB1, DB2, DB3 and DB4 databases using the original minutiae data (N), 20\% missing minutiae (M) and $20 \%$ spurious minutiae (S).
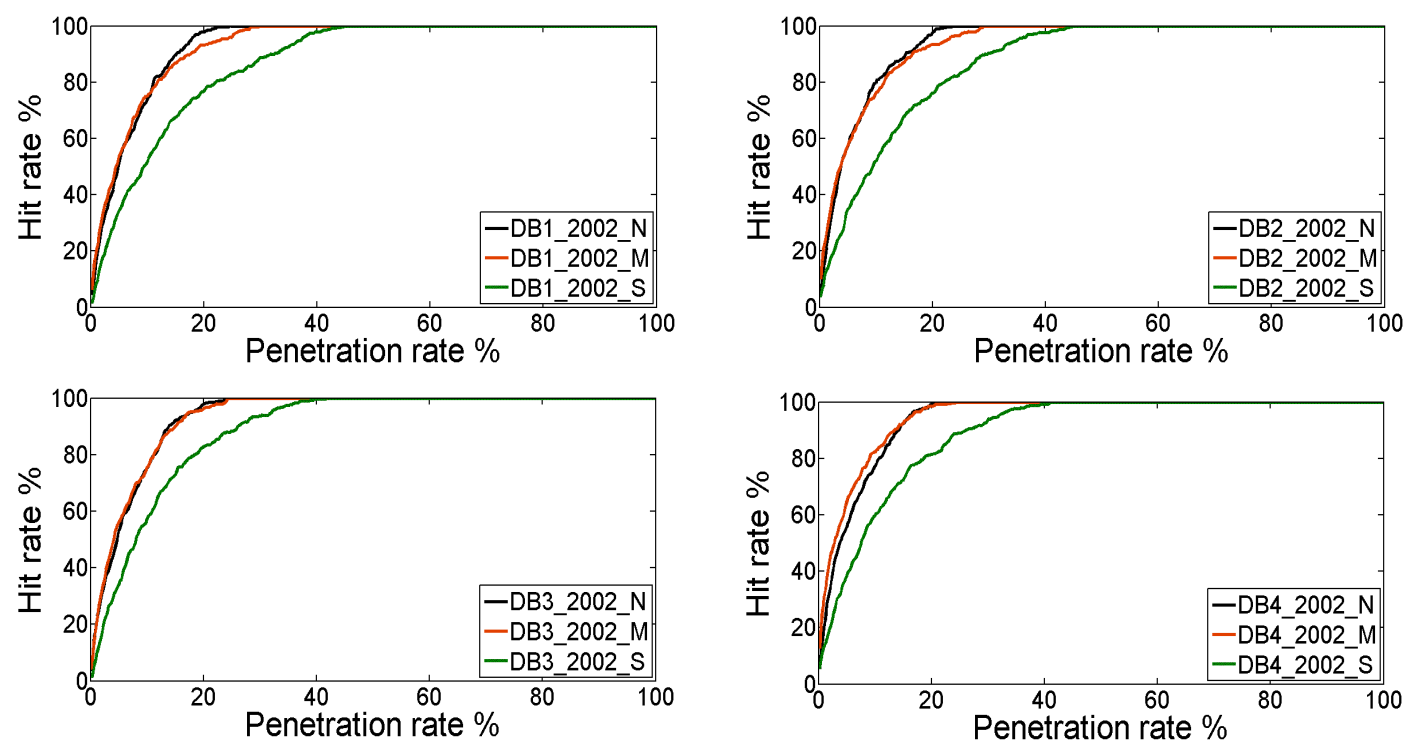

Figure 7: Performance on FVC 2002 DB1, DB2, DB3 and DB4 databases using the original minutiae data (N), 20\% missing minutiae (M) and $20 \%$ spurious minutiae (S). 

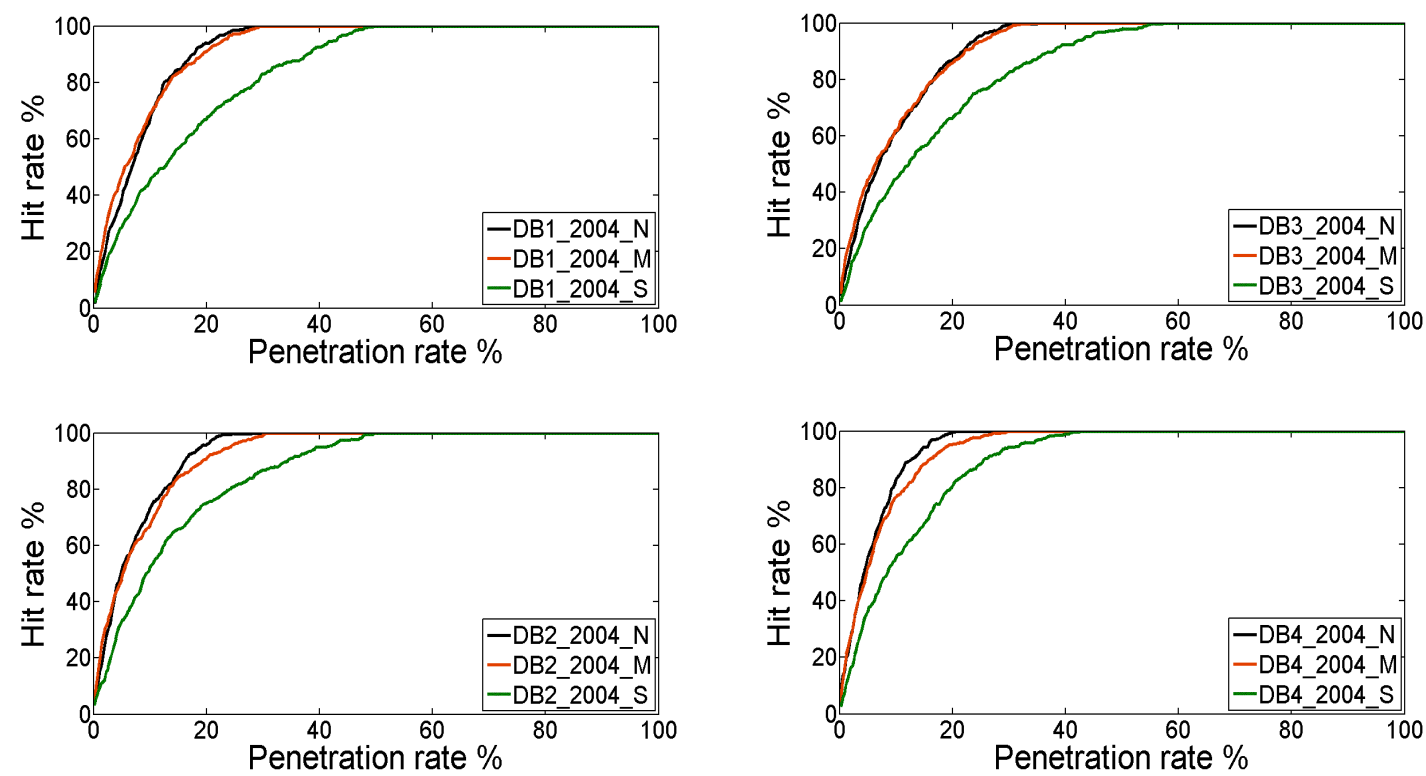

Figure 8: Performance on FVC 2004 DB1, DB2, DB3 and DB4 databases using the original minutiae data (N), 20\% missing minutiae (M) and $20 \%$ spurious minutiae (S).

Table 2: Average penetration rates when using Minutiae Quadruplets, Low-order Delaunay Triangle (LoD) [7] and Minutiae Triplets [6] at hit rates of $99 \%$ and $100 \%$. The evaluation protocol was based on [7].

\begin{tabular}{|l|r|r|r|r|}
\hline & \multicolumn{2}{|c|}{$99 \%$ hit rate } & \multicolumn{2}{c|}{$100 \%$ hit rate } \\
\hline FVC databases (DB1) & \multicolumn{1}{|c|}{2002} & \multicolumn{1}{c|}{2004} & 2002 & \multicolumn{1}{c|}{2004} \\
\hline Minutiae Quadruplets & $11.2 \%$ & $11.8 \%$ & $\mathbf{1 1 . 8 \%}$ & $\mathbf{1 2 . 0 \%}$ \\
\hline Low-order Delaunay Triangle [7] & $\mathbf{8 . 1 \%}$ & $\mathbf{1 0 . 0 \%}$ & $18.1 \%$ & $20.9 \%$ \\
\hline Minutiae Triplets [7] (based on [6]) & $23.6 \%$ & $27.2 \%$ & $38.1 \%$ & $40.9 \%$ \\
\hline
\end{tabular}

\section{Comparison of Minutiae Quadruplets with Other Fingerprint Indexing Techniques}

The proposed technique is compared with three other fingerprint indexing techniques; Minutiae triplets [6], Low-order Delaunay triangle [7] and Composite sets of reduced SIFT features [14].

The proposed technique based on quadruplets was evaluated on FVC 2002 DB1 and 2004 DB1, and compared with Liang et al.'s [7] results on minutiae triplets and low-order Delaunay triangles. For this comparison, we follow the testing scenario of Liang et al [7] and use the first three images for each subject as gallery images and the rest as probes. Table 2 shows the average penetration rates of minutiae triplets, low-order Delaunay triangle [7] and minutiae quadruplets at $99 \%$ and $100 \%$ hit rates.

The proposed technique was also compared with the method based on composite sets of reduced SIFT features [14]. For this comparison on the FVC 2000 DB2 database, the first image of each subject was enrolled in the gallery and the rest of the images were used as probes, as done in [14].

Table 3 shows the average penetration rate of Shuai et al.'s method [14] compared with minutiae quadruplets at a $100 \%$ hit rate.

Table 3: Average penetration rates when using Minutiae Quadruplets and SIFT features on FVC 2000 DB2. The evaluation protocol was based on [14].

\begin{tabular}{|c|c|c|}
\hline & $99 \%$ hit rate & $100 \%$ hit rate \\
\hline Minutiae Quadruplets & $\mathbf{1 9 \%}$ & $\mathbf{2 6 \%}$ \\
\hline SIFT Features [14] & $21 \%$ & $91 \%$ \\
\hline
\end{tabular}

In Table 3, the average penetration at a hit rate of $100 \%$ is $26.33 \%$ for Minutiae Quadruplets and 91\% for SIFT Features. 


\section{Conclusions}

In this paper, the use of minutiae quadruplets has been proposed for indexing fingerprints. The consistent performance of the proposed method on the FVC 2000, 2002 and 2004 databases (set A) indicates that the proposed technique is database-independent. Experiments on fingerprints with spurious minutiae points and fingerprints with missing minutiae show that the technique is reasonably robust. The retrieval strategy is computationally inexpensive and the proposed method has small storage requirements. Further analysis is necessary to leverage the proposed technique into operational systems. Based on the experiments conducted in this work, it is apparent that minutiae quadruplets are a viable alternative to minutiae triplets for indexing.

\section{Acknowledgments}

This work was done when Iloanusi was a Visiting Scholar at West Virginia University. Iloanusi was supported by grants from the University of Nigeria, Nsukka. Gyaourova and Ross were supported by US NSF CAREER grant number IIS 0642554.

\section{References}

[1] D. Maltoni, D. Mario, A.K. Jain and S. Prabhakar. Handbook of Fingerprint Recognition. 2nd Ed. SpringerVerlag London Limited, pp 236 - 262, 2009.

[2] A. Ross and R. Mukherjee. Augmenting Ridge Curves with Minutiae Triplets for Fingerprint Indexing. In: Proc. of SPIE Conference on Biometric Technology for Human Identification IV, Orlando, USA. April 2007.

[3] J. Feng and A. Cai. Fingerprint Indexing using Ridge Invariants. In: Proc. of the 18th International Conference on Pattern Recognition, 2006.

[4] J. Li, W. Yau and H. Wang. Fingerprint Indexing Based on Symmetrical Measurement. In: Proc of the $18^{\text {th }}$ International Conference on Pattern Recognition, 2006.

[5] R. S. Germain, A. Califano and S. Colville. Fingerprint Matching using Transformation Parameter Clustering. IEEE Computational Science and Engineering. pp. 42-49, 1997.

[6] B. Bhanu and X. Tan. Fingerprint Indexing Based on Novel Features of Minutiae Triplets. IEEE Transactions on Pattern Analysis and Machine Intelligence. 25(5): 616622, 2003.

[7] X. Liang, A. Bishnu and T. Asano. A Robust Fingerprint Indexing Scheme Using Minutia Neighborhood Structure and Low-Order Delaunay Triangles. IEEE Transactions on Information Forensics and Security, 2(4):721-733, 2007.

[8] J. Boer, A. M. Bazen and S. H. Gerez. Indexing Fingerprint Databases Based on Multiple Features. In: ProRISC 2001 Workshop on Circuits, Systems and Signal Processing, 2001.

[9] A. Gyaourova and A. Ross. A Novel Coding Scheme for Indexing Fingerprint Patterns. In: Proc. Joint IAPR
International Workshop on Structural, Syntactic, and Statistical Pattern Recognition. Springer-Verlag, pp. 755 764, 2008.

[10] A.K. Jain. Data Clustering: 50 Years Beyond K-Means. Pattern Recognition Letters, 31(8): 651-666, 2010.

[11] D. Maio, D. Maltoni, R. J. Cappelli, L. Wayman and A. K. Jain, "FVC2000: Fingerprint Verification Competition". In: Proc. 15th International Conference Pattern Recognition, Barcelona, September 3-8, 2000.

[12] D. Maio, D. Maltoni, R. J. Cappelli, L. Wayman and A. K. Jain, "FVC2002: Second Fingerprint Verification Competition", Proc. International Conference on Pattern Recognition, Quebec City, August 11-15, pp. 811-814, 2002.

[13] D. Maio, D. Maltoni, R. J. Cappelli, L. Wayman and A. K. Jain, "FVC2004: Third Fingerprint Verification Competition", Proc. International Conference on Biometric Authentication (ICBA), Hong Kong, pp. 1-7, July 2004.

[14] X. Shuai, C. Zhang and P. Hao. Fingerprint Indexing Based on Composite Set of Reduced SIFT Features, In: IEEE International Conference on Pattern Recognition (ICPR), Tampa, FL, USA, December 8-11, pp. 1-4, 2008. 